Note

\title{
THE SMALLEST NONEVASIVE GRAPH PROPERTY
}

\author{
Michą AdAmaszeK \\ Institute of Mathematics, University of Bremen \\ Bibliothekstr. 1 \\ 28359 Bremen, Germany \\ e-mail: aszek@mimuw.edu.pl
}

\begin{abstract}
A property of $n$-vertex graphs is called evasive if every algorithm testing this property by asking questions of the form "is there an edge between vertices $u$ and $v$ " requires, in the worst case, to ask about all pairs of vertices. Most "natural" graph properties are either evasive or conjectured to be such, and of the few examples of nontrivial nonevasive properties scattered in the literature the smallest one has $n=6$.

We exhibit a nontrivial, nonevasive property of 5 -vertex graphs and show that it is essentially the unique such with $n \leq 5$.
\end{abstract}

Keywords: graph properties, evasiveness, complexity.

2010 Mathematics Subject Classification: 05C99, 00A08.

\section{REFERENCES}

[1] M.R. Best, P. van Emde Boas and H.W. Lenstra, A sharpened version of the Aanderaa-Rosenberg conjecture, Afd. Zuivere Wisk. 30/74 (1974).

[2] C.E. Chronaki, A survey of evasivness: lower bounds on decision-tree complexity of Boolean functions, The University of Rochester Tech. Report (1990), available from www.ics.forth.gr $/{ }^{\sim}$ chronaki/papers/ur/eve.ps.

[3] J. Kahn, M. Saks and D. Sturtevant, A topological approach to evasiveness, Combinatorica 4 (1984) 249-315.

doi:10.1007/BF02579140

[4] D. Kozlov, Combinatorial Algebraic Topology (Algorithms and Computation in Mathematics, Vol. 21, Springer-Verlag Berlin Heidelberg, 2008).

[5] M. de Longueville, A Course in Topological Combinatorics (Universitext, Springer New York, 2013). 
[6] L. Lovász and N. Young, Lecture Notes on Evasiveness of Graph Properties, Tech. Rep. CS-TR-317-91, Computer Science Dept., Princeton University, available from arxiv/0205031.

[7] E.C. Milner and D.J.A. Welsh, On the computational complexity of graph theoretical properties, Proc. 5th British Comb. Conf. Aberdeen 1975 (1976) 471-487.

[8] The On-Line Encyclopedia of Integer Sequences, published electronically at oeis.org.

[9] V. Welker, Constructions preserving evasiveness and collapsibility, Discrete Math. 207 (1999) 243-255.

doi:10.1016/S0012-365X(99)00049-7

Received 6 August 2013

Revised 6 November 2013

Accepted 6 November 2013 